\title{
INNOVACIÓN PÚBLICA EN AMÉRICA LATINA: CONCEPTOS, EXPERIENCIAS EXITOSAS, DESAFÍOS Y OBSTÁCULOS
}

\author{
Freddy Mariñez NaVARro \\ El Colegio de Jalisco, México \\ freddy.marinez@coljal.edu.mx
}

\section{RESUMEN}

De acuerdo a algunas miradas en el debate académico, la innovación pública es posible con una plataforma abierta de gobierno democrático que implique gobernanza colaborativa, espacios de participación digitales y presenciales, liderazgo público innovador, redes de capacidades e innovación social. En este sentido, la innovación pública quiere decir ir más allá de lo gubernamental. Implica un compromiso cívico que se expresa en términos colaborativos entre ciudadanos, funcionarios públicos y sector privado, que apunta a producir cambios en valores, actitudes, procesos y liderazgos para la co-creación de decisiones y políticas públicas que logren el mejoramiento del aprovisionamiento de los servicios públicos.Este artículo discute las diversas aproximaciones que existen en la literatura actual sobre el tema, analiza los artículos que forman parte del dossier que Revista de Gestión Pública ha preparado sobre innovación pública en América Latina,e identifica una serie de cuestiones críticas tanto para el debate académico como para el impulso de la innovación en las administraciones públicas latinoamericanas.

Palabras clave: Innovación en el sector público, Innovación pública, Gestión pública colaborativa, América Latina. 


\title{
PUBLIC INNOVATION IN LATIN AMERICA: CONCEPTS, SUCCESSFUL EXPERIENCES, CHALLENGES AND OBSTACLES
}

\begin{abstract}
According to certain perspectives within academic debate, public innovation is possible with an open platform of democratic government which implies collaborative governance, spaces for digital and in-person participation, innovative public leadership, networks of capacities and social innovation. In this respect, public innovation means going beyond the governmental. It implies a civic commitment which is expressed in collaborative terms among citizens, civil servants and the private sector, and which aims to produce changes in values, attitudes, processes and leaderships for the cocreation of decisions and public policies which achieve the improvement of the provision of public services. This article discusses the different approaches to be found in the current literature on the subject, analyzes the articles that form part of the dossier which the Revista de Gestion Pública prepared on public innovation in Latin America, and identifies a series of questions critical both for academic debate as well as for the boosting of innovation in Latin American public administrations.
\end{abstract}

Keywords: Innovation in the public sector, Public innovation, Collaborative public management, Latin America. 


\section{PUNTO INICIAL DE CONTEXTO}

De acuerdo a algunas miradas en el debate académico, la innovación pública es posible con una plataforma abierta de gobierno democrático que implique gobernanza colaborativa, espacios de participación digitales y presenciales, liderazgo público innovador, redes de capacidades e innovación social. En este sentido, la innovación pública quiere decir ir más allá de lo gubernamental. Implica un compromiso cívico que se expresa en términos colaborativos entre ciudadanos, funcionarios públicos y sector privado, que apunta a producir cambios en valores, actitudes, procesos y liderazgos para la co-creación de decisiones y políticas públicas que logren el mejoramiento del aprovisionamiento de los servicios públicos. Es importante dejar claro que los espacios de la innovación pública los vemos en cuatro ámbitos: políticas públicas, administración pública como gobernanza colaborativa, gobierno relacional (gobierno abierto) y organizaciones públicas ciudadanas.

Revista de Gestión Pública ha preparado un dossier sobre este tema, el cual busca generar una reflexión sobre el concepto de innovación pública a la luz de los avances y debates conceptuales. De igual forma, este dossier tiene una fuerte orientación hacia la presentación de trabajos de investigación que sean capaces de presentar evidencia empírica sobre innovación pública en el contexto latinoamericano. Temas de especial interés son abordados en la perspectiva teórica como empírica a través de estudios de casos, tales como las diferencias y semejanzas de la innovación pública y la innovación gubernamental; las instituciones fundamentales para la innovación pública; los diseños organizativos y de procesos para una innovación abierta (open collaboration); la innovación en las políticas públicas; los flujos de comunicación en la administración pública sustentada en la colaboración; el gobierno abierto como innovación pública y los procesos colaborativos y de co-creación en las organizaciones de participación pública.

\section{LA INNOVACIÓN PÚBLICA COMO DESAFÍO}

Como sabemos, la eficacia y la eficiencia de las organizaciones públicas se han venido buscando a través de la mejora de la calidad y de la innovación gubernamental sustentado en los siguientes pilares de la calidad total: la satisfacción de los clientes, la mejora continua de los procesos y el desarrollo del personal. La aplicación de este concepto dio como resultado el reacomodo del arreglo tradicional de las organizaciones, induciendo cambios como el privilegio de los resultados financieros de las empresas y de los gobiernos, desplazándose hacia la calidad de los productos, enfatizándose en el sector público, en la eficiencia económica de las 
operaciones y el equilibrio riguroso de las finanzas públicas; también hizo énfasis en la productividad, moviendo la atención de los productos hacia los clientes, generando que el producto sea un componente integrado del servicio, elevándose la productividad a la dimensión de competitividad por la calidad de los productos. Por otro lado, vemos que la administración hacia adentro, que caracterizó a la administración weberiana, se ha extrovertido hacia los usuarios de los bienes y servicios de la organización, poniendo el punto final de una administración pública sin público y un gobierno sin ciudadanos (Aguilar Villanueva 2006).

A diferencia del concepto de innovación gerencial explicado, partimos de un concepto de innovación donde el centro del análisis es el compromiso de los ciudadanos con el gobierno, facilitando la inteligencia cívica y colectiva, el mejoramiento del control de la gestión vía la contraloría social, así como una concepción compartida de lo público entendida como competencias de colaboración y co-creación para el logro legitimado de resultados públicos.

En esta dirección, presentamos la innovación pública atendiendo a una premisa y a dos direcciones. La premisa es que no puede haber innovación pública sin un sector público innovador y una ciudadanía innovadora. En cuanto a las direcciones, la primera de ellas no es más que los arreglos de los ciudadanos, actores gubernamentales y no gubernamentales que permiten identificar problemáticas públicas, así como herramientas y dispositivos innovadores de co-creación para el logro de respuestas y soluciones nuevas en el espacio de lo público presencial y digital. Y la otra dirección, consecuencia de la anterior, a las modalidades democráticas de articulación de estos actores en la organización pública, produciendo una nueva configuración entre el Estado y la sociedad que asegura el aprendizaje colectivo y los saberes cívicos necesarios para una co-creación de lo público. Solo así vemos a las organizaciones públicas más relacionales, a la gestión pública más participativa y a las políticas públicas, en todo su ciclo, más deliberativas en la construcción de la agenda, más incluyentes en aportar soluciones (diseño), y más colaborativas por los múltiples actores en acción en la implementación

Para hablar de innovación pública, entonces, tendríamos que referirnos primeramente al concepto de la innovación en el sector público.

La innovación en el sector público por lo general, implica nuevos métodos de entrega de los servicios públicos. En ocasiones, el sector público desarrolla nuevos programas, que podía pensarse en ellos como análoga a los nuevos productos del sector privado (Borins 2012: 
$5)$.

Es por ello que la innovación en este sector, ya sea en términos de cómo los servicios públicos deben ser entregados, o en la creación de infraestructura social que puede utilizarse de forma novedosa, toma tiempo ya que son cambios en los procesos, impactos y resultados (Borins 2012)

El mismo Borins (2002) nos introduce tres tipos de innovaciones en el sector público. El primer tipo es el de "abajo hacia arriba" que se traduce cuando alguien tiene una buena idea y la coloca en el buzón de sugerencias, dándole seguimiento la organización ejecutora a través de sus canales normales. El segundo tipo de innovación se relaciona con las repuestas a las crisis, ya que no son más que resultados de factores que son impredecibles o externos. Y por último, la innovación vinculada con las reestructuraciones organizativas dirigidas por jefes de agencias. En este sentido, la restructuración es una repuesta a la organización del sector público que simplemente no está cumpliendo con las expectativas normales.

Por otro lado, Potts y Kastelle (citados por Casebourne 2014), nos introducen tres razones clave del porqué de la Innovación en el sector público.

- Tamaño del sector público: el sector público impacta en muchos países en el porcentaje del PIB. La innovación en el sector público puede afectar el crecimiento general de la productividad reduciendo el costo de los insumos, el aumento del valor de los productos, mediante una mejor organización.

- Necesidad de la política para que coincida con la evolución de las economías en el marco de la globalización.

- El sector público establece las reglas del juego para la innovación del sector privado.

La innovación en el sector público presenta varios enfoques que es importante visualizarlos con el objetivo de ver sus diferencias y semejanzas en cuanto a sus planteamientos (Gow 2014). Everett Rogers es considerado el decano de la innovación en el sector público. Su libro titulado Diffusion of Innovations del año 2001, logra contribuir en la difusión de la innovación en los casos de comunicación. Por otro lado está Sanford Borins quien sustenta un rico trabajo sobre las innovaciones gubernamentales en los Estado Unidos en la perspectiva holística. Su principal libro del año 1998 se titula Innovations With Integraty. How Local Heroes Are Transforming Anmerican Government. Otra perspectiva es la desarrollada por Eleanor 
Glor quien es fundadora y editora de The Innovation Journal: The Public Sector Innovation Journal. Ella, desde el año 2001, ha venido sistematizando el cómo los estudios de la innovación pueden ser vistos como parte de la teoría organizacional, la ciencia de la gestión, el aprendizaje social y el análisis de sistemas. Involucra el enfoque de la teoría inductiva, construida sobre la base de la experiencia y la literatura.

Para los efectos de este dossier, es importante aclarar que innovar en lo público (innovación pública), quiere decir ir más allá de lo gubernamental (innovación en el sector público). Implica compromiso cívico, que se expresa en términos colaborativos entre los funcionarios públicos, el sector privado, los ciudadanos y las organizaciones civiles para la co-creación de métodos, técnicas y habilidades, haciendo uso de las tecnologías de información y comunicación (Web 2.0). Vale la pena dejar claro que esta innovación debe apuntar a producir cambios en valores, actitudes, procesos y liderazgos, que contribuyen a generar valor público. Es decir, una parte importante de lo que hace el sector público es prestar servicios públicos relativamente estables. La innovación pública, complementando, apuesta a una relación de colaboración entre actores gubernamentales y no gubernamentales para el impulso de decisiones y políticas públicas que logren el mejoramiento del aprovisionamiento de los servicios públicos con nuevas soluciones. En otras palabras, tal como lo expresa Criado, Rojas y Silván en este dossier, "no solo hay un nuevo modelo de producción de servicios públicos detrás, sino una nueva forma de innovar”. En la Tabla 1 podemos observar las diferencias entre estos dos tipos de innovaciones, la pública y en el sector público. 
Tabla 1: Innovación en el sector público e Innovación pública

\begin{tabular}{|c|c|c|}
\hline Características & $\begin{array}{l}\text { Innovación en el } \\
\text { sector público }\end{array}$ & Innovación Pública \\
\hline Tipos de apoyo & $\begin{array}{l}\text { La cooperación } \\
\text { de todos los } \\
\text { funcionarios } \\
\text { públicos para el } \\
\text { cambio de los } \\
\text { procesos. }\end{array}$ & $\begin{array}{l}\text { Los funcionarios públicos de todas las } \\
\text { agencias en colaboración con actores no } \\
\text { gubernamentales logran cambios en los } \\
\text { procesos de la organización, mediante la } \\
\text { co-creación }\end{array}$ \\
\hline $\begin{array}{l}\text { Dirección de la } \\
\text { innovación }\end{array}$ & $\begin{array}{c}\text { La reorganización } \\
\text { de la } \\
\text { administración } \\
\text { para el cambio } \\
\text { organizacional es } \\
\text { de arriba hacia } \\
\text { abajo (top-down) }\end{array}$ & $\begin{array}{l}\text { La reorganización de la administración } \\
\text { es producto de la colaboración (open } \\
\text { collaboration) entre distintos actores } \\
\text { (externos e internos). Tendencia a la } \\
\text { organización relacional y horizontal }\end{array}$ \\
\hline Tipo de relación & $\begin{array}{l}\text { Cambios en las } \\
\text { formas de relación. } \\
\text { Comunicación } \\
\text { en línea entre } \\
\text { funcionarios } \\
\text { públicos y usuarios } \\
\text { y ciudadanos } \\
\text { de una forma } \\
\text { unidireccional. } \\
\text { Plataformas } \\
\text { de consultas } \\
\text { ciudadanas. }\end{array}$ & $\begin{array}{l}\text { Las formas de relación están sustentada } \\
\text { en: peer production, open source, open } \\
\text { collaboration y el crowdsurcing }\end{array}$ \\
\hline $\begin{array}{l}\text { Tipos de } \\
\text { creación de } \\
\text { valor }\end{array}$ & $\begin{array}{l}\text { Valor público } \\
\text { producido por el } \\
\text { gobierno }\end{array}$ & $\begin{array}{l}\text { Valor público producido por la colaboración } \\
\text { (co-creación) entre el gobierno, ciudadanos y } \\
\text { sector privado. }\end{array}$ \\
\hline
\end{tabular}

Herramientas tecnológicas

Web 1.0

Web 2.0

Fuente: Elaboración propia a partir deMariñez Navarro (2015).

Los cuatro conceptos clave que han impulsado el fenómeno Web 2.0 y la innovación pública y en consecuencia el trabajo de co-creación son los siguientes (Mergel, Schweik, Fountain 2009): primeramente el peer production (producción entre iguales), que describe un especial tipo de sistema de producción donde los individuos actúan en respuestas a sus propias necesidades e intereses y de manera descentralizada. Otro atributo, además de que los usuarios recurran para hacer cosas que le interesan, es que los esfuerzos se desarrollan en crowd (multitud) y en público. El segundo concepto es el open source and open content del copyright al copyleft que provee al usuario el derecho a copiar, modificar y distribuir nuevos derivados del Software. Pero los mandatos que se obtienen de estos 
derivados tienen la condición igual que el software original. Se refiere al open source, software (libre), que no es más que la colaboración como la "instancia de producción", quintaesencia de la producción entre iguales (commons-based peer). El open source depende de muchas contribuciones individuales para un proyecto común. Compartir sus contribuciones y con una variedad de motivaciones sin derechos a ser excluidos de la comunidad digital (Benkler, citado por Mergel, Schweik, Fountain 2009). El tercer concepto es el user-centered innovation que primeramente demostró que los usuarios más desarrolladores son las empresas privadas, los gobiernos y las organizaciones de ciudadanos, convencidos que el Open Source es un juego que conduce a un cambio significativo en la composición del ecosistema del código abierto. Y segundo, la mayoría del software producido es desarrollado por programadores que son usuarios del Software. La idea de los usuarios como innovadores, es que añaden significativamente los incentivos que impulsan a la gente a contribuir con calidad. La existencia de colaboración de códigos abiertos como "redes de innovación centradas en el usuario", es quizás una sorpresa para muchos en el momento en que los innovadores revelan libremente sus innovaciones.

El cuarto concepto es el crowdsourcing o inteligencia colectiva. Si tomamos la definición de Howe (citado por Mergel, Schweik, Fountain 2009), el crowdsourcing es el acto de tomar un trabajo tradicionalmente desempeńado por un agente designado (usualmente un empleado) y la externalización generalmente a un grupo grande e indefinido de personas en forma de una convocatoria abierta y conectados. Las conexiones de masas como tal se aprovechan a través de Internet con el fin de adquirir recursos, conocimiento, experiencia o tiempo (colaboración abierta). Algunas de las ventajas del crowdsourcing son las siguientes: 1) compilación de una gran variedad de propuestas de alta calidad por parte de la comunidad; 2) disminución de costes; 3) feedback interno y permanente y; 4) generación continua de ideas innovadoras. En otras palabras, esta inteligencia colectiva asociada a las nuevas tecnologías sociales es un proceso de producción de servicios que permite que frente a la existencia de un problema, o una necesidad de colaboración en la construcción de una información pública, la organización selecciona a un colectivo; la comunidad se implica en la coproducción de la información o en la resolución conjunta de la tarea; y el resultado dispone de un beneficio dual y mutuo para la organización y la sabiduría colectiva (Linders, Criado y Villodre y Sørensen y Torfing, citados en este dossier por Criado, Rojas y Silván).

Los casos de innovación pública referidos en este dossier, tales como el Centro de Integración Ciudadana en México (Rubio Campos y Calzada Torres) y Novagob.lab-UAM (Criado, Rojas y Silván) se sustentan en 
los avances dentro de las dinámicas del Crowdsourcing impulsando nuevos modelos de servicio público donde la colaboración está presente en la solución a problemas públicos. En el caso de San Pedro Garza García, México es la utilización de los diferentes tipos de crowdsourcing (crowdwisdom, crowdcreation, crowdfunding y crownvoting), lo que genera la articulación entre ciudadanos y gobiernos para la creación de reportes geo referenciados en temas como situaciones de riesgo, emergencias, seguridad pública, vialidad, tránsito así como servicios públicos.

\section{El caso de Novagob.lab-UAM,}

[...] pretende ser una síntesis de las mejores ideas observadas en el ámbito internacional, si bien el modelo que se propone aquí se encuentra más cercano al GovLab: pretende ser una institución de innovación mediante el diseño de prototipos para el sector público creando entornos de experimentación controlada, capacitar a los empleados públicos para que creen valor público mediante la innovación y atraer a innovadores públicos (una filosofía que dio inicio a NovaGob como red social de referencia del sector público en Iberoamérica) para aliarse con gobiernos y administraciones en el diseño y mejora de políticas públicas orientadas a la satisfacción de los intereses y derechos de los ciudadanos.

La experiencia de desarrollo de la innovación en el sector público chileno, presentada en esta publicación por Arros Valdivia y Ramírez-Alujas, es un fenómeno reciente y concebido como más allá de la modernización de la gestión pública. Creado en el 2014, el Laboratorio de Gobierno es considerado como una iniciativa puesta en marcha por el Gobierno de Chile y se compone de un equipo multidisciplinar de siete ministerios. Se centra sobre todo en la ayuda en el diseño de servicios públicos y en la formación a sus empleados para reorientar las políticas a los intereses de los ciudadanos mediante la aplicación de métodos innovadores. Se ha destacado en el ámbito de las políticas sanitarias a través de la iniciativa Impacta Salud (también liderada por el Gobierno de Chile). Estos autores afirman que el proceso de establecer la innovación como una herramienta permanente e inserta dentro de los paradigmas imperantes implica el logro de diversas variables altamente complejas que requieren del aporte, compromiso y dedicación de una serie de actores. En este contexto, el Laboratorio de Gobierno, se posiciona como la institucionalidad encargada de impulsar y promover la innovación, y en este sentido, se observa que este contribuye a ser la primera institucionalidad que permite comenzar a transformar los paradigmas existentes, y quien comienza a forjar el camino para que la innovación pueda instalarse como práctica permanente. Esto no 
implica que no se requiera en el futuro revisar si es suficiente la existencia de un Laboratorio de Gobierno o se requieran además otras estrategias que permitan que la innovación se transforme en una práctica permanente.

El caso que nos presenta Juan Carlos Montero ("Innovación Pública para la Seguridad. El caso de Nuevo León, México”) tomó en consideración varias aristas de la innovación. Primero como un producto organizacional que implica la ruptura entre paradigmas; segundo, la innovación es también producto de la cooperación y la suma de esfuerzos de los distintos actores; tercero, la innovación es vista como un proceso sistemático, no excepcional, lo cual requiere de una transformación organizacional que lo permita y de agentes promotores que la impulsen y conduzcan, considerando que si esto no se logra, la innovación tenderá a ser obsoleta y a que los recursos invertidos en su desarrollo constituyan un desperdicio; y por último, la diferenciación de los conceptos de innovación gubernamental y de innovación pública, considerando al primero como una transformación hacia dentro del gobierno y al segundo orientado a la transformación en las relaciones del gobierno con la sociedad.

También el gobierno abierto se ha venido viendo como innovación pública. Definido también como Collaborative Public Management (McGuire 2009), Citizen Engagement (Hickle 2008, OECD 2004), Wiki Government (Noveck 2009), Public Sector Clients (Alford 2009); el gobierno abierto nos brinda múltiples definiciones y en cada una se identifican tendencias con un abordaje teórico muy particular. En este sentido, hemos sistematizado las distintas propuestas conceptuales de este tipo de organización pública. Una primera definición que ha tenido mucho eco en el mundo académico y gubernamental es el que aborda el gobierno en la perspectiva o como resultado del cambio tecnológico donde la Web 2.0 juega un rol fundamental y el centro de su análisis son las Tecnologías de información y Comunicación -TICs- (Nan 2012, Harrison 2011, Noveck 2009, 2010, Center for Tecnhology in Government de la University of Albany y el Open Government Working Group del Gobierno de los Estados Unidos de América). Una segunda definición enfatiza la importancia de concebir el gobierno abierto como un cambio en los valores en la organización y sus procesos, en síntesis, se traduce en una nueva cultura organizacional (Calderón y Lorenzo 2010, Ramírez Alujas 2011). La tercera definición con la que se ha identificado el gobierno abierto es aquella que se explica a través del open data, o mejor conocido como datos abiertos, y que viene a revolucionar el concepto de transparencia tal como se había venido implementando (Open Government Partnership, 2011, Fung, Graham y Weil 2007, Bouhadana y Gilles 2014, Dawes 2010). Hemos visualizado una cuarta definición donde el gobierno abre la 
posibilidad de la participación de los ciudadanos para monitorear e influir los procesos de gobierno a través del acceso a la información y el acceso a la arena de la hechura de las decisiones y de las políticas (Meijer, Curtin y Hillebrandt 2012, Guardián Orta 2010, Calderón y Lorenzo 2010).

Todas estas definiciones tienden a identificar, evaluar y caracterizar diferentes formas de integración ciudadana en el sector público vista por la vía del marco de la innovación en la transparencia y el acceso a la información, así como en la colaboración y la participación, haciendo uso de las tecnologías de información y comunicación. Con una visión crítica, Hevia de la Jara y Arturo Durán Padilla nos muestran un panorama de reflexión interesante con el caso de los gobiernos abiertos en entornos analógicos a nivel municipal y estatal en México, precisando más en el estado Veracruz.

Los resultados generales alcanzados por los autores en este ejercicio sugieren la necesidad de pensar cómo crear una agenda de estrategias alternativas que permita, a las iniciativas ciudadanas en contextos analógicos, transitar o escalar hacia etapas que posibiliten aprovechar las potencialidades de la apertura gubernamental. En este sentido, los autores consideran la pertinencia de reflexionar los contenidos de una agenda de gobierno abierto en torno a tres dimensiones, dado que Veracruz constituye la tercera entidad más poblada de México. Además, por sus condiciones socioeconómicas, es una de las regiones con mayor pobreza y desigualdad de acuerdo a las más recientes mediciones, y sus condiciones políticas están marcadas por administraciones estatales de baja calidad y capacidad, prevaleciendo una profunda crisis social, de derechos humanos y seguridad. La primera dimensión la constituye una relación técnica compuesta de las condiciones de recursos y cobertura de redes para la transmisión de información pública. La segunda se vincula a una estructura jurídico administrativa de la vida local ante la incorporación de nuevas racionalidades normativas. Mientras la tercera dimensión tiene que ver con la esfera de la política tradicional.

Para discutir la dimensión técnica cabe anticipar que, en la sustitución de pautas de usabilidad analógica, existen profundas dificultades. La promesa del empleo de mecanismos técnicos está dirigida a garantizar mayor eficiencia de los recursos empleados. Sin embrago, frente a los propósitos por obtener resultados eficientes bajo la operación de plataformas de servicios automatizados, el paradigma fundado en la dependencia tecnológica implica la instalación de un sistema de mecanismos complejos. 
No obstante esta tendencia de la innovación pública, muchos desafíos observamos cuando revisamos experiencias en este sentido. Pedersen (2013), por ejemplo, nos alerta argumentando que el éxito de la innovación está relacionado con el grado en la que toda la organización está dirigida a la innovación, y no una parte de ella. Esto lo lleva a plantearse las siguientes interrogantes: ¿qué tipo de organizaciones públicas son innovadoras y quiénes son los conductores de las innovaciones en las organizaciones públicas?, ¿cómo el sector público innova las organizaciones y cómo acceden y usan el nuevo conocimiento generado en la co-producción y cocreación?, ¿qué funcionarios públicos requiere la administración pública para estructurar, organizar, promover e implementar la innovación pública? Son los grandes desafíos de esta tendencia innovadora, al menos en los países de América Latina donde las administraciones públicas y gobiernos portan aún rasgos de patrimonialismo, falta de profesionalización, ausencia de modernización tecnológica y opacidad. 


\section{REFERENCIAS}

Aguilar Villanueva, L. F. (2006). Gobernanza y Gestión Pública. México: Fondo de Cultura Económica.

Alford, J. (2009). Public Sector Clients: From Service-Delivery to Coproduction. Basingstoke: Palgrave Macmillan.

Borins, S. (2002). Leadership and Innovation in the Public Sector. Leadership \& Organization Development Journal, 23 (8), 467-476.

. (2012). Innovation in Business and Government: Looking Forward. Canberra: Australian National University.

Bouhadana, I. y Gilles, W. (2014). Droit et Gouvernance des Administrations Publiques à l Ère du Numérique. París: Les editions IMDEV.

Calderon, C. y Lorenzo, S. (Coords.). (2010). Open Government. Gobierno Abierto. Madrid: Algón Editores.

Casebourne, J. (2014). Why Motivation Matters in Public Sector Innovation. Londres: Nesta.

Dawes, S. (2010). Stewardship and Usefulness: Policy Principles for Information-based Transparency. Governement Information Quarterly, 27 (4), 377-383.

Fung, Archon, Mary Graham and David Weil (2007). Full Disclosure: The Perils and Promise of Transparency Cambridge University Press.

Guardián Orta, C. (2010). ¿Transparencia? Calderon, C. y Lorenzo, S. (Coords.), Open Government. Gobierrno Abierto. Madrid: Algón Editores.

Harrison, T., Santiago Guerrero, Burk, G. y Cook, M. (2011). Open Government and E-Government: Democratic Challenges from a Public Value Perspective. En The Proceeding of the 12th Annual International Digital Governement Research Conference.

Hickley, M. (2008). The Grounds for Citizen Engagement and the Roles of Planners. Verlag: VDM.

Gow, J. (2014). Public Sector Innovation Theory Revisited. The Innovation Journal. The Public Sector Innovation Journal, 10 (2), 1-22. 
Mariñez Navarro, Freddy (2015). Innovación Pública. Para que Funcionarios Públicos y Ciudadanos Actúen con Saberes Cívicos. México: Fontamara.

McGuire, M. (2009). Collaborative Public Management: Assessing What We Know and How We Know It. Public Administration Review, 66 (1), 33-43.

Meijer, A., Curtin, D. y Hillebrandt, M. (2012). Open government: Connecting Vision and Voice. International Review of Administrative Sciences, 78 (1), 10-29.

Mergel, I., Schweik, C. y Fountain, J. (2009). The Transformational Effect of Web 2.0 Technologies on Government. Disponible en: http://ssrn. com/abstract=1412796 [01-06-2017].

Nan, T. (2012). 'Citizens' Attitudes toward Open Government and Government 2.0. International Review of Administrative Sciences, 78 (2), 346-368.

Noveck, B. (2009). Wiki Government: How Technology Can Make Government Better, Democracy Stronger, and Citizens More Powerful. Washington: Brookings Institute Press.

. (2010). The Single Point of Failure. En Lathrop, D. y Ruma, L. (Eds.), Open Government. Collaboration, Transparency, and Participation in Practice. Sebastopol: O'Reilly.

Open Government Partnership. (2011). Open Government Declaration. Disponible en http://www.opengovpartnership.org/about/opengovernment-declaration [18-06-2017].

Organización para la Cooperación y el Desarrollo Económico (OECD). (2004). Promise and Problems of E-Democracy: Challenge of Online Citizen Engagement. París: OECD Publications

Pedersen, R. (2013). Collaborative Narrative Innovation. A Case of Public Innovation in Denmark. En Müller, A. y Becker, L. (Eds.), Narrative and Innnovation. Management-Culture-Interpretation. Wiesbaden: Springer.

Ramírez Alujas, A. (2011). Gobierno Abierto y Modernización de la Gestión Pública: Tendencias Actuales y el (Inevitable) Camino que Viene. Reflexiones seminales. Revista Enfoques, IX (15), 99-125.

Recibido: 10-05-2017

Aceptación de la versión final: 31-05-2017 